\title{
ARTICLE OPEN Electron engineering of metallic multiferroic polarons in epitaxial $\mathrm{BaTiO}_{3}$
}

\author{
Tao Xu $\mathbb{D}^{1,2}$, Takahiro Shimada ${ }^{2}$, Yasumitsu Araki ${ }^{2}$, Masataka Mori ${ }^{2}$, Gen Fujimoto ${ }^{2}$, Jie Wang ${ }^{3}$, Tong-Yi Zhang ${ }^{1}$ and Takayuki Kitamura ${ }^{2}$
}

The coexistence of ferroelectricity, conductivity, and magnetism in a single-phase material has attracted considerable attention due to fundamental interest and tremendous technological potential. However, their mutually exclusive mechanisms hinder the discovery of multifunctional conducting multiferroics. Here, we propose a new material design approach for electron engineering to enable these conflicting properties to coexist. We use first principles calculations to demonstrate that appropriate mechanical strain can turn the excess electrons in doped $\mathrm{BaTiO}_{3}$ from a free-carrier configuration to a localized polaronic state by modulating the electron-phonon coupling. The resulting localized spin-polarized electron survives the host ferroelectricity and consequently manifests as a multiferroic polaron. The multiferroic properties coexist with the electronic conductivity arising from the highhopping mobility of the polaron, which enables the doped epitaxial $\mathrm{BaTiO}_{3}$ to act as a multiferroic conducting material. This mechanical control over the electron configuration is a potential path toward unusual coexisting properties.

npj Computational Materials (2019)5:23; https://doi.org/10.1038/s41524-019-0163-6

\section{INTRODUCTION}

The quest for the coexistence of normally conflicting states in a single material has been one of the most fundamental goals of condensed matter science as a playground of nontrivial physical phenomena and unconventional multifunctional materials. ${ }^{1-3}$ Particularly, the complex and subtle interplays of the charge, spin, and elastic degrees of freedom in a ferroic system have recently attracted an explosion of interest because of the fascinating physical properties and manifold possibilities of new technological paradigms such as multiple-state memory, ${ }^{4}$ spintronics, ${ }^{5}$ and photovoltaics. ${ }^{6}$ However, single-phase multifunctional materials, which have these collective characteristics of ferroelectricity, conductivity, and magnetism, are extremely rare. This is because the conduction electrons in material screen the long-range Coulomb interactions that are responsible for ferroelectric instability and thus destroy the ferroelectric distortion. ${ }^{7}$ Furthermore, the number of single-phase multiferroics is limited, although great progress has been made to obtain new magnetic ferroelectrics. $^{8-10}$ Recently, electron-doped ferroelectrics, which are obtained via chemical substitution or oxygen vacancy, have been suggested as a promising candidate for metallic ferroelectrics $^{11,12}$ or even multiferroics. However, the free carriers inevitably quench the ferroelectricity at high density, since the conventional contraindication between them is still not circumvented in these materials. Therefore, a new concept based on a unique mechanism instead of simple electron doping is desired to obtain truly metallic multiferroic properties, which will also provide significant insight into diverse functionalities in perovskite oxides.

Because electrons are of key importance in determining the electronic, magnetic, and optical properties of those functional materials with competing physical phenomena due to their association with the spin and lattice degrees of freedom, ${ }^{13-17}$ the manipulation and control over the behavior of charge carriers have been recognized as an effective avenue to discover exotic electronic phenomena and new technology breakthroughs beyond the conventional physical laws. One of the most striking manifestations of the electron configuration is the formation of highly confined two-dimensional electron gas, which has been proven to produce various unique behaviors such as the interfacial metallic phase $\mathrm{e}^{18,19}$ and the coexistence of ferromagnetism and superconductivity. ${ }^{20}$ Other low-dimensional structurally confined electron configurations have also been demonstrated to introduce new quantum phenomena, ${ }^{21}$ tunable electronic properties, ${ }^{22}$ and enhanced Coulomb interactions. ${ }^{23}$ Therefore, the controllability of the electron configuration, especially confined in reduced dimensionality, offer fertile ground for unusual coexisting states and extraordinary functionalities that are inaccessible in host materials.

Here, we demonstrate a new approach to control the electron behavior by strain engineering instead of the common structuraldriven confinement while achieving the unusual multiferroic conducting properties in electron-doped $\mathrm{BaTiO}_{3}$. The excess electrons are in the common free-carrier states in this traditional ferroelectric material, whereas an appropriate mechanical strain unexpectedly turns the delocalized electrons into the self-trapped polaronic state. The localized excess electrons in a spin-polarized polaronic state do not suppress the ferroelectricity and induce the multiferroic properties. Therefore, the multiferroic properties combined with electronic conductivity, which arises from the high-hopping mobility of the polaron, enable the doped epitaxial $\mathrm{BaTiO}_{3}$ to act as a multiferroic conducting material.

\footnotetext{
${ }^{1}$ Materials Genome Institute, Shanghai University, Shanghai Materials Genome Institute, Shanghai 200444, China; ${ }^{2}$ Department of Mechanical Engineering and Science, Kyoto

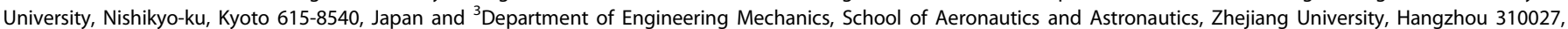
China

Correspondence: Tao Xu (xutao6313@shu.edu.cn) or Takahiro Shimada (shimada@me.kyoto-u.ac.jp)
}

Received: 4 June 2018 Accepted: 23 January 2019

Published online: 20 February 2019 
(a)

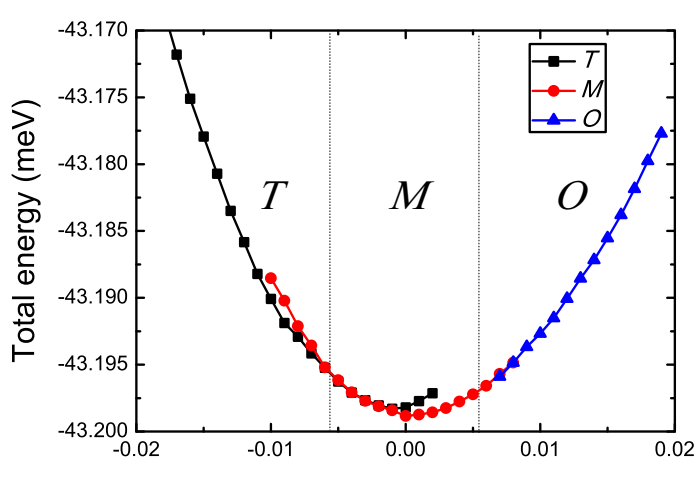

(001) in-plane strain

(c)
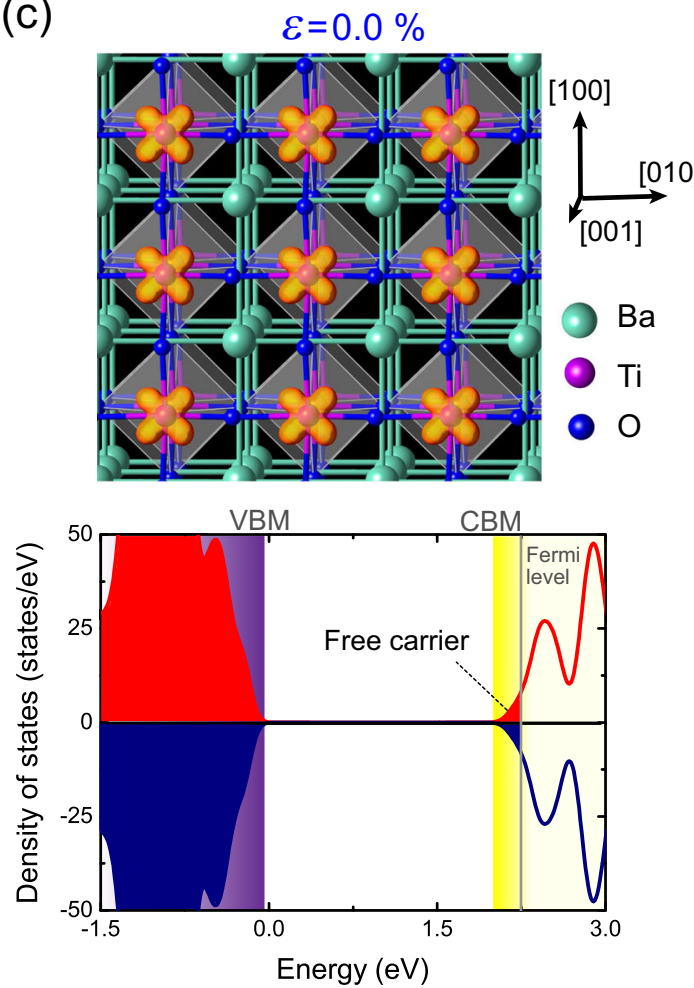

Free-carrier (b)

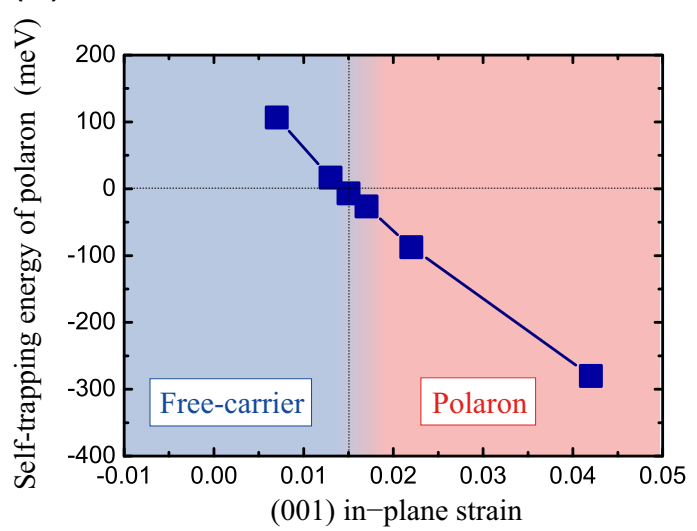

(d)
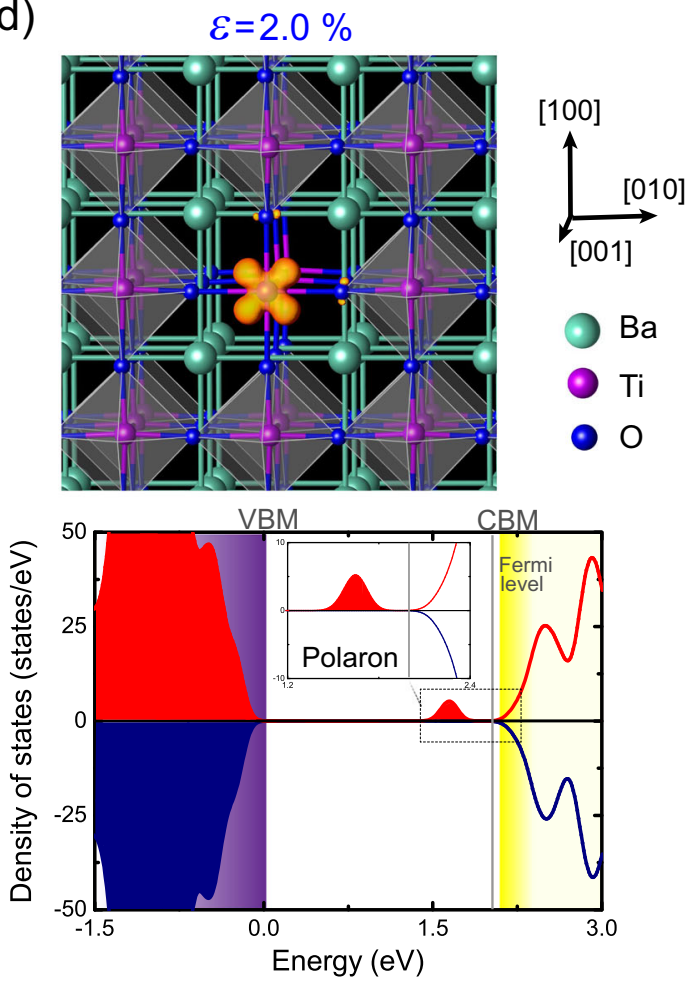

Polaron

Fig. 1 Stable configuration of excess electron in $\mathrm{BaTiO}_{3}$ under the (001) in-plane strain. a Evolution of the self-trapping energy of the polaron with respect to the epitaxial strain. $\mathbf{b}$ Electronic properties of free carrier in $\mathrm{BaTiO}_{3}$ with zero strain. The orange isosurfaces represent the upspin densities of $0.0005 \AA^{-3}$. The zero energy is set to the valence-band maximum (VBM). CBM denotes the conduction-band minimum. The Fermi level is denoted by the vertical silver line. c Electronic properties of electron polaron in $\mathrm{BaTiO}_{3}$ with $2.0 \%$ strain. The orange isosurfaces represent the up-spin densities of $0.005 \AA^{-3}$

\section{RESULTS AND DISCUSSION}

The epitaxial strain dependence of the total energy of various $\mathrm{BaTiO}_{3}$ crystal structures is illustrated in Fig. 1a. In the considered range of epitaxial strains, $\mathrm{BaTiO}_{3}$ displays the tetragonal $T$, monoclinic $M$ and orthorhombic $O$ phases, which undergo the transitions of $T-M$ at $\varepsilon=-0.6 \%$ and $M-O$ at $\varepsilon=0.6 \%$. These crystallographic phases are consistent with previous investigations. ${ }^{24,25}$ Then, we probe the stable configurations of the excess electrons in epitaxial (001) $\mathrm{BaTiO}_{3}$ by calculating the self-trapping energy $E_{\text {form }}$ of the polaron, which is defined as the energy difference between the polaronic $\left(E_{\mathrm{p}}\right)$ and free-carrier states $\left(E_{\mathrm{f}}\right)$, i.e., $E_{\text {form }}=E_{\mathrm{p}}-E_{\mathrm{f}}$. The evolution of the self-trapping energy of the polaron with respect to the epitaxial strain is depicted in Fig. $1 \mathrm{~b}$. In the strain-free state, the formation of polaronic states is unfavorable, and thus, the excess electrons are in the delocalized free-carrier states. As visualized from the squared wave functions in Fig. 1c, these excess electrons homogeneously spread over the entire crystal of the symmetrically equivalent $\mathrm{Ti}$ sites with the upand down-spin states being separately delocalized over neighboring layers. The electronic density of states clearly demonstrates free-carrier states, since the Fermi levels cross over the conduction bands with populated conducting states (Fig. 1c). Similarly, the excess electrons behave as delocalized free carriers in the structures under strains smaller than $1.5 \%$.

However, $E_{\text {form }}$ decreases to almost zero at $\varepsilon=1.5 \%$. Furthermore, when the misfit strain increases to $1.7 \%, E_{\text {form }}$ becomes 
negative $(-26.3 \mathrm{meV})$, which indicates the energetic favor of the polaronic states with respect to their delocalized counterparts and the spontaneous formation of electron polarons in this mechanical state. The spatial charge distribution of the polaron (at $\varepsilon=2.0 \%$ ) is similarly shown in Fig. 1d. The excess electrons are evidently trapped at one $\mathrm{Ti}$ site with a small contribution from the neighboring $\mathrm{O}$ atoms and are coherently coupled to an obvious local lattice distortion. The formation of the localized electron induces an occupied isolated state as visualized from the electronic density of state. The bandgap of the polaron system is estimated to be $0.40 \mathrm{eV}$. Therefore, the misfit strain effectively turns the excess electrons from the free-carrier configuration into a self-trapped state in the form of small polarons in $\mathrm{BaTiO}_{3}$. The polaronic character of the excess electron is also observed with further applications of the tensile strain, and the polaronic state becomes significantly more stable with increasing strain. Thus, the excess electrons undergo the delocalized-to-localized electronic state transition at a critical strain of approximately $\varepsilon=1.5 \%$. These results suggest that the misfit strain plays a vital role in the confinement of electrons and formation of a polaronic state. A similar type of localized excess charge (hole polaron) is also obtained in other perovskite oxides. ${ }^{26-28}$

The delocalized-to-localized transition of the electron configuration arises from the competition between the loss in strain energy $\left(E_{\mathrm{ST}}\right)$, which is associated with the lattice distortion, and the electronic energy gained from the electron localization $\left(E_{\mathrm{EL}}\right)$ in the polaron formation process. ${ }^{29} E_{\mathrm{EL}}$ is insensitive to the role of the strain, whereas $E_{\mathrm{ST}}$ decreases in the tensile condition due to the increased Ti-O bonds. This phenomenon can be clearly understood by tracking the phonon mode involved with the polaron formation through the electron-polaron interaction. As visualized

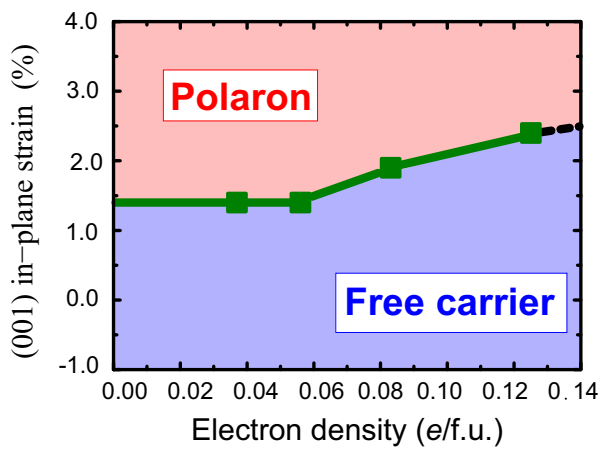

Fig. 2 Stability region of polaron and free-carrier states in electrondoped $\mathrm{BaTiO}_{3}$ with different doping densities and mechanical strains

(a)

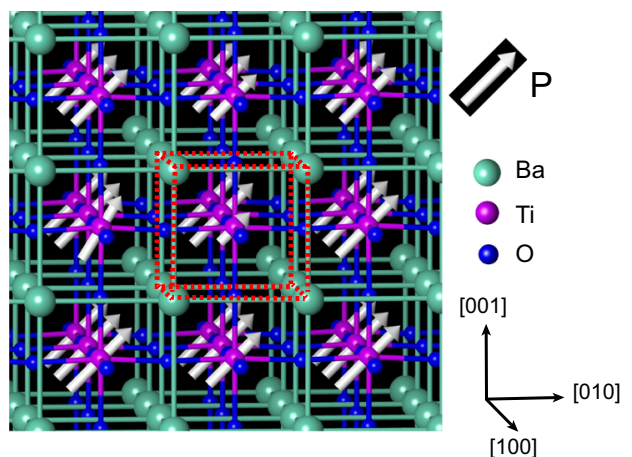

in Fig. S4, the breathing (oxygen bond-stretching) phonon mode associated with the polaronic distortion is softened when the strain increases, which indicates the greater tendency of the system toward this structural distortion. As a result, the electron-phonon coupling is increased, and the loss in strain energy $\left(E_{\mathrm{ST}}\right)$ due to the polaron formation is reduced. Therefore, the energy gain is larger than the energy loss, and the formation of the polaronic state becomes energetically favorable in the tensile-strained structure with strain larger than $1.5 \%$. We further investigate the strain dependences of the values of $\left(1 / \varepsilon_{\infty}-1 / \varepsilon_{0}\right)$ in $\mathrm{BaTiO}_{3}$, where $\varepsilon_{\infty}$ and $\varepsilon_{0}$ are the optical and static dielectric constants. This quantity is closely related to Fröhlich constant ${ }^{30}$ and is a measure of the electron-phonon coupling strength. As shown in Figure S6, the calculated values increase with increasing strain, which also accounts for the polaron stabilization by tensile strains.

It is instructive to further ascertain the role of carrier density on the behavior of excess electrons, which is realized by performing simulations in the same manner using supercells with different sizes. According to our calculations, the critical strain for the transition of free-carrier and polaron configurations increases with increasing density. Specifically, the critical strain rise to $2.4 \%$ when the doping density reaches 0.125 e/f.u., which indicates that the stability of the polaron decreases when the electron density increases. All of the above results show that a stable polaron regime in terms of strain and electron density can be established, as illustrated in Fig. 2.

The effect of these different electron configurations on the ferroelectricity in $\mathrm{BaTiO}_{3}$ is quantified by calculating the ferroelectric distortion, which is defined as the relative displacement of $\mathrm{Ti}$ and $\mathrm{O}$. The ferroelectric polarization are intrinsically incompatible with excess free-carrier electrons due to the screening of electrostatic interactions that are responsible for ferroelectricity, and inevitably vanish at high-electron concentration, as demonstrated in previous studies ${ }^{31-33}$ (e.g., 0.085 e/f.u. in $\mathrm{BaTiO}_{3}{ }^{33}$ ). In contrast, as visualized in Fig. $3 a$ for the polaronic states, the ferroelectric distortion in the $\mathrm{BaTiO}_{3}$ system is only marginally affected by the excess electrons, except for the local suppression of ferroelectricity on the unit cell that accommodates the electron polaron. The ferroelectric distortion persists at all the high electron doping densities applied here. The strain dependence of the average polar distortion is shown in the Supplementary Information (Fig. S3). The observed polar distortion is small at small applied strains and sharply increases when the strain is larger than $1.0 \%$. We also calculate the variation of polar distortions in $\mathrm{BaTiO}_{3}$ with respect to the doping electron density at several strain states (Fig. S4). The results show that the polar distortions decrease with increasing electronic density but generally increase with

(b)

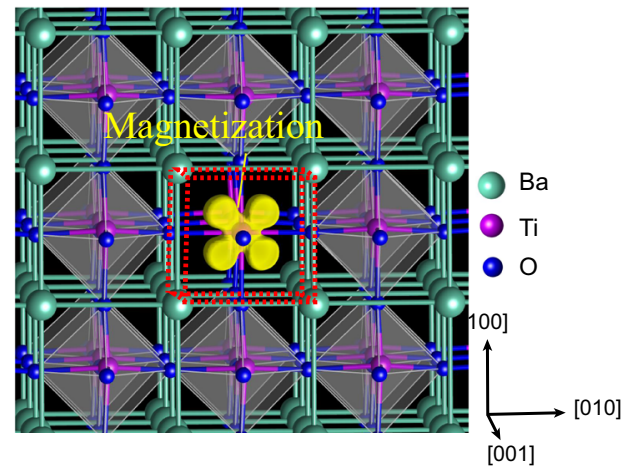

Fig. 3 Multiferroic properties in electron-doped $\mathrm{BaTiO}_{3}$. The red cubic box is the unit cell that accommodates the electron polaron. a Local ferroelectric polarization in each unit cell. The white arrow $\mathrm{P}$ indicates the corresponding ferroelectric polarization of $\mathrm{BaTiO}_{3}$ without electron doping. b Magnetic spin-density distribution. The yellow area represents the isosurfaces of spin densities of $0.01 \mu_{\mathrm{B}} \AA^{-3}$ 
increasing strain. Because of the formation of polarons, the doping density has a relatively small effect on the polaron distortions at a strain of $4.0 \%$. It is worth to mention that defects (e.g., oxygen

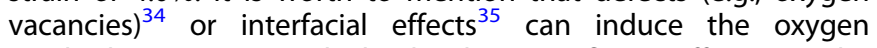
octahedron rotation, which also has significant effects on the ferroelectric properties. However, the rotation is not observed in the present study.

The amplitude of the ferroelectric polarization was further quantified using Berry phase approach. ${ }^{36}$ The result shows that the average polarization is $(0.26,0.26,0) \mathrm{C} / \mathrm{cm}^{2}$ in the polaron system at a strain of $1.5 \%$, which is close to the value in bulk $\mathrm{BaTiO}_{3} .{ }^{37}$ We further calculated the energy difference of the polaronic states in the ferroelectric and paraelectric phases and obtained approximately $26 \mathrm{meV} / \mathrm{f}$.u., which is also comparable to that of bulk $\mathrm{BaTiO}_{3}(18 \mathrm{meV} / \mathrm{f} . u$. $) .^{38}$ This result suggests an easy transition between the two states and the switchability of the polarization in the system. A direct application of an electric field to the doped materials may be difficult to switch the polarization, since the polaron system has certain conductivity, and the excess electrons in such system may become mobile. The ferroelectric displacement switching can be realized by constructing a superlattice that is sufficiently resistive to sustain the coercive voltage, as proposed for some polar metals. ${ }^{32,39}$ In addition, Hao et al. ${ }^{29}$ showed that the polarons in perovskite oxides move via hopping and lead to the conductivity at high temperature, while they get trapped and the system may be insulating or semiconducting when temperature decreases. So, the switching is possible at low temperature due to trapped polaronic states. Therefore, the ferroelectric properties are sustained and coexist with the doped electrons in the $\mathrm{BaTiO}_{3}$ system through the confinement of free carriers.

Figure $3 \mathrm{~b}$ shows the magnetic spin-density distribution associated with these excess electrons in the polaronic states. Despite being intrinsically nonmagnetic, a nontrivial magnetic moment of $1.0 \mu_{B}$ rapidly appears in the polaronic states in the $\mathrm{BaTiO}_{3}$ system when the misfit strain is larger than $1.5 \%$. The emerging magnetic moment is positive and highly concentrated on the atomic-scale region of the $\mathrm{Ti}$ site, which signals the appearance of magnetism in the doped $\mathrm{BaTiO}_{3}$. This highly confined atomic-scale magnetism is attributed to the localized polaronic state in the bandgap, which reduces the normal $\mathrm{Ti}^{4+}$ to $\mathrm{Ti}^{3+}$ and creates the surplus unpaired $\mathrm{Ti} 3 d$ spin-polarized electrons, as discussed regarding the electronic density. Therefore, the confinement of free carriers through the strain engineering manifests as multiferroic polarons in electron-doped $\mathrm{BaTiO}_{3}$. The coupling of the local magnetic moments is found to have a ferromagnetic order by introducing two polarons separated by a distance of approximately $7.04 \AA$, which is the largest possible distance in the supercell. The ferromagnetic state is more stable than the nonmagnetic one with an energy difference of $113 \mathrm{meV}$, while the antiferromagtic state is unstable and spontaneously relaxes to the ferromagnetic state. This strong ferromagnetic exchange coupling compared with that in room-temperature ferromagnetism ${ }^{40}$ suggests that the ferromagnetic order in the present system may also persist at room temperature through the accumulation of polarons. Since the doped excess electrons carry their own spins unchanged when they wander from atom to atom, the coupling via the electron polarons will decrease the energy of the system when the spins are pointing in the same direction, which creates the ferromagnetic order in the system. We also performed the calculation with two polarons localized on the same $\mathrm{Ti}$ in the same supercell and found that this configuration was unstable in the $\mathrm{BaTiO}_{3}$ system.

To fathom the dynamics and associated conductivity of the multiferroic polarons, we further investigated the mobility of electron polarons in term of their activation barrier. The barrier height for a polaron hopping between two neighboring Ti sites is estimated by linearly interpolating two polaron configurations

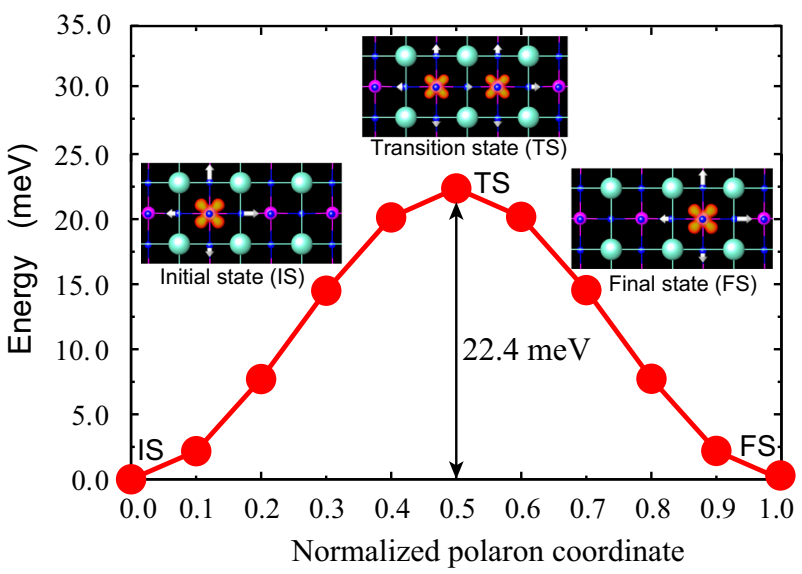

Fig. 4 Energy variation for the polaron migration path between two minimum-energy states (initial state (IS) and final state (FS)). The insets show the electron densities with an isosurface value of $0.006 \AA^{-3}$

(see Methods section) using the state-of-the-art hybrid functional. ${ }^{41,42}$ The calculated energy barriers in Fig. 4 show that the multiferroic polaron is highly activated with activation energy $E_{a}$ of only $22.4 \mathrm{meV}$. This value is in the same order as the experimentally obtained value $(68 \mathrm{meV})^{43}$ and much smaller than those in $\mathrm{CaTiO}_{3}$ and other oxides (e.g., near $300 \mathrm{meV}$ in $\mathrm{TiO}_{2}$ ). ${ }^{44-49}$ We also note that the nudge elastic band method ${ }^{27}$ may obtain a smaller energy barrier, which indicates the weak trapping effect of the polaron states and high mobile characteristics of polarons in $\mathrm{BaTiO}_{3}$. The high mobility of electron polarons causes electrical charge transport and polaronic conductivity, which can be estimated in the adiabatic small-polaron hopping mechanism as: $^{50-52} \sigma=\left[\sigma_{0} / T\right]\left[\exp \left(-E_{a} / k_{B} T\right)\right]$. Here, $\sigma_{0}$ is a pre-exponential factor; ${ }^{52,53} k_{\mathrm{B}}$ and $T$ are Boltzmann's constant and the absolute temperature, respectively. Taking the values of the calculated energy barrier, we obtain the polaronic conductivity of approximately $76.2 \mathrm{~S} / \mathrm{cm}$ at room temperature $(300 \mathrm{~K})$, which is intermediate between those in normal semiconductors and metals. ${ }^{54}$ Such small-polaron hopping conductivity has also been observed in many oxide materials, ${ }^{55-60}$ although the value is lower because of the higher energy barrier for polaron hopping with respect to the corresponding value predicted here in $\mathrm{BaTiO}_{3}$. Therefore, the electron-doped epitaxial $\mathrm{BaTiO}_{3}$ displays a level of electronic conductivity as a result of the electron confinement and highpolaron migration. Experimentally, the excess electrons can be introduced by optical methods ${ }^{61}$ or electric-field effect ${ }^{62}$ without pinning centers for charges to obtain the proposed polaronic conductivity.

The above results demonstrate the unusual coexistence of ferroelectricity, magnetism, and conductivity in electron-doped $\mathrm{BaTiO}_{3}$ via the strain-engineered modulation of electron configurations. The excess electrons in the free-carrier states inevitably suppress the ferroelectricity because of the screening of the Coulomb interaction; ${ }^{63,64}$ while the formation of the spin-polarized polaron by strain confines the excess electrons to the atomic-scale site and maintains the ferroelectric properties of the system even at a sufficient large doping density. These multiferroic polarons in conjunction with the electronic conductivity due to their highhopping mobility enable the doped epitaxial $\mathrm{BaTiO}_{3}$ to act as multiferroic conducting material. This new design strategy based on the electron manipulation circumvents the conventional restrictions between ferroelectricity and conductivity and between ferroelectric and magnetic properties, which provides a method to synthesize new multiferroic semiconductors. This new class of multifunctional materials with integrated multiferroic and conductive properties open fascinating perspectives for innovative 
applications such as magnetoelectricity and photovoltaics. It is worth to mention that the polar distortion can coexist with metallicity in some other electron-doped materials such as $\mathrm{PbTiO}_{3}{ }^{65}$ whereas the mechanism (the origin of ferroelectricity) in these materials is the lone-pair mechanism and meta-screening effect instead of the present one.

Our demonstrations also illustrate the concept of electron engineering not by chemical modification or structural confinement but by mechanical strain, which provides a possibility in the search and design of new functionalities. The application of this concept is not specific to the particular epitaxial ferroelectric system that is described here, but general to a range of complex structures and artificial geometries with intrinsic mechanical strain, such as the grain boundary and interface. It has been reported that the self-trapped energy of small hole polaron is largely reduced at a ferroelectric domain wall very recently, ${ }^{66}$ which may attribute to the mechanism that we propose here. Therefore, present study suggests a tempting prospect to achieve widely tunable electron configurations, including electron chains and two-dimensional electron gas, which will also provide a rich playground for the exploration of much more intriguing phenomena.

In summary, a new material design concept for electron engineering is proposed, which provides a possibility for the unusual coexistence of the ferroelectricity, magnetism, and conductivity in a single-phase material. The mechanical strain provides a viable means to modulate the shape and configuration of electrons through the modulation of electron-phonon coupling and results in the multiferroic polaron in electron-doped $\mathrm{BaTiO}_{3}$. These multiferroic properties further coexist with the electronic conductivity, which originates from the intense mobility of electron polarons. Therefore, the deliberate mechanical control over the electron configuration may become a promising paradigm for unusual coexisting properties and new technology breakthroughs.

\section{METHODS}

First-principles calculations are performed using density functional theory $(D F T)+U$ approach $^{67}$ implemented in the VASP code. ${ }^{68,69}$ The $3 \times 3 \times 3$ Monkhorst-Pack k-point mesh ${ }^{70}$ is used for the Brillouin zone integrations. The $k$-point sampling was chosen by the convergence tests in Figure S2. In all calculations, the electronic convergence tolerance was set to $1.0 \times$ $10-6 \mathrm{eV} / \mathrm{cell}$, and the atomic positions were fully relaxed until Hellamnn-Feynman force was below $0.01 \mathrm{eV} / \mathrm{A}$. The Coulomb and exchange parameters $U$ and $J(U=3.925 \mathrm{eV}$ and $J=0.511 \mathrm{eV})$ are calculated fully $a b$ initio from the constrained random phase approximation. ${ }^{71}$ The DFT $+\mathrm{U}$ method has been highly successful in reproducing the electronic properties of electron-doped systems ${ }^{27,29,66,72}$ at minimal added computational cost. The common functionals, including the local density approximation and generalized gradient approximation, usually lead to artificial electron delocalization and fail to reproduce the polaron formation. $^{28,72}$ Although the state-of-the-art Heyd-Scuseria-Ernzerhof (HSE06) screened hybrid functional ${ }^{41,42}$ can more accurately describe the electronic structures of various semiconductors, exhaustive calculations of the strain and density dependence of the polaron stability using a hybrid functional is computationally exceedingly prohibitive. Therefore, we only use the HSEO6 to cross-check the stability of the polaron obtained from the $\mathrm{DFT}+\mathrm{U}$ method. The electron-doped $\mathrm{BaTiO}_{3}$ systems are modeled by the common supercell approach, which comprises $3 \times 3 \times 3$ perovskite unit cells. Since the effect of the finite charge concentration is necessary to investigate the stable configuration of excess charge, Makov-Payne correction, ${ }^{73}$ which excludes the effect of the neighboring polaron and charge concentration, is not applied in the present study. The phonon dispersion was calculated by computing the dynamical matrix using a finite displacement method, where each ion was displaced by $0.01 \AA$. Atomic and structural relaxations are performed by constraining the inplane lattice parameters of the supercell $\boldsymbol{a}_{1}$ and $\boldsymbol{a}_{2}$ to simulate the (001) epitaxial condition while allowing the remainder of out-of-plane lattice, $\boldsymbol{a}_{3}$, to fully relax during the calculations. The intrinsic electron behaviors in the ferroelectrics are investigated by injecting one electron in the calculated supercell with the same amount of homogeneous positive background charges, which correspond to a density of 0.037 e/f.u., and subsequently relaxing the atomic positions at each epitaxial condition. Both free-carrier configuration and polaronic state, which consist of electrons and a small concomitant lattice distortion, are considered (Fig. S1). The free-carrier configuration is obtained by relaxing the initial structure from the perfect $\mathrm{BaTiO}_{3}$ geometry, and the polaronic state develops when an expansion of the oxygen octahedron around a chosen Ti atom is applied in the starting geometry.

A linear interpolation scheme $\mathrm{e}^{74,75}$ is used to calculate the polaron migration pathway $C$, as $C=t \times C 1+(1-t) \times C 2$. Here, $C 1$ and $C 2$ are the coordinates of all atoms in the initial and final polaron configurations, respectively; $t$ is varied in the range of $0-1$.

The pre-exponential factor ${ }^{76} \sigma_{0}$ in the estimation of polaronic conductivity is given by

$\sigma_{0}=\frac{g c N(1-c) e^{2} a^{2} \nu}{\kappa}$,

where $g$ is a geometrical factor, $c$ is the fraction of sites occupied by polaron, $N$ is the density of conducting sites, $a$ is the hopping distance, i.e., the distance of two neighboring $\mathrm{Ti}$ atoms, and $v$ is the lattice vibrational frequency.

\section{DATA AVAILABILITY}

All relevant data are available from the author.

\section{ACKNOWLEDGMENTS}

The authors acknowledge the financial support for T.X. by the National Natural Science Foundation of China (Grant no. 11802169), for T.S. and T.K. by JSPS KAKENH (25000012, 26289006, 15K13831, and 17H03145), for J.W. by the Fundamental Research Funds for the Central Universities 2018XZZX001-05, and for T.Y.Z. by the 111 project (No. D16002) from the State Administration of Foreign Experts Affairs and the Ministry of Education, PRC.

\section{AUTHOR CONTRIBUTIONS}

T.X. and T.S. conceived the project, designed, and directed the computational experiments, and wrote the entire manuscript. Y.A., M.M. and G.F. performed the theoretical calculations and interpreted the data. J.W. and T.Y.Z. supported the calculations and discussed the results. T.K. supervised the work and provided critical feedback on the manuscript. All authors read and commented on the manuscript. T.X. and T.S. equally contributed to this work.

\section{ADDITIONAL INFORMATION}

Supplementary information accompanies the paper on the npj Computational Materials website (https://doi.org/10.1038/s41524-019-0163-6).

Competing interests: The authors declare no competing interests.

Publisher's note: Springer Nature remains neutral with regard to jurisdictional claims in published maps and institutional affiliations.

\section{REFERENCES}

1. Birgeneau, M. A. R. J., Shirane, G. \& Endoh, Y. Magnetic, transport, and optical properties of monolayer copper oxides. Rev. Mod. Phys. 70, 897-928 (1998).

2. Hill, N. A. \& Filippetti, A. Why are there any magnetic ferroelectrics? J. Magn. Magn. Mater. 242, 976-979 (2002).

3. Anderson, P. W. \& Blount, E. I. Symmetry considerations on martensitic transformations: "ferroelectric" metals? Phys. Rev. Lett. 14, 217-219 (1965).

4. Béa, H., Gajek, M., Bibes, M. \& Barthélémy, A. Spintronics with multiferroics. J. Phys. Condens. Matter 20, 434221 (2008).

5. Wolf, S. A. et al. Spintronics: a spin-based electronics vision for the future. Science 294, 1488 (2001).

6. Yang, S. Y. et al. Above-bandgap voltages from ferroelectric photovoltaic devices. Nat. Nanotech. 5, 143 (2010).

7. Lines, M. E. \& Glass, A. M. Principles and Applications of Ferroelectrics and Related Materials (Oxford University Press, Oxford, 2001).

8. Fennie, C. J. \& Rabe, K. M. Magnetic and electric phase control in epitaxial EuTiO from first principles. Phys. Rev. Lett. 97, 267602 (2006). 
9. Lee, J. H. et al. A strong ferroelectric ferromagnet created by means of spin-lattice coupling. Nature 466, 954 (2010).

10. Lee, J. H. \& Rabe, K. M. Epitaxial-strain-induced multiferroicity in $\mathrm{SrMnO}_{3}$ from first principles. Phys. Rev. Lett. 104, 207204 (2010)

11. Fujioka, J. et al. Ferroelectric-like metallic state in electron doped $\mathrm{BaTiO}_{3}$. Sci. Rep. 5, 13207 (2015).

12. Jeong, I.-K. et al. Structural evolution across the insulator-metal transition in oxygen-deficient $\mathrm{BaTiO}_{3-\delta}$ studied using neutron total scattering and rietveld analysis. Phys. Rev. B 84, 064125 (2011).

13. Dai, P., Hu, J. \& Dagotto, E. Magnetism and its microscopic origin in iron-based high-temperature superconductors. Nat. Phys. 8, 709-718 (2012).

14. Eerenstein, W., Mathur, N. D. \& Scott, J. F. Multiferroic and magnetoelectric materials. Nature 442, 759-765 (2006).

15. Xu, T., Shimada, T., Araki, Y., Wang, J. \& Kitamura, T. Multiferroic domain walls in ferroelectric PbTiO3 with oxygen deficiency. Nano Lett. 16, 454 (2016).

16. Hwang, J., Kolodiazhnyi, T., Yang, J. \& Couillard, M. Doping and temperaturedependent optical properties of oxygen-reduced $\mathrm{BaTiO}_{3-\delta}$. Phys. Rev. B 82, 4982-4985 (2010).

17. Yu, S., Zhang, J., Tang, Y. \& Ouyang, M. Engineering acoustic phonons and electron-phonon coupling by the nanoscale interface. Nano Lett. 15, 6282-6288 (2015).

18. Jang, J. et al. Observation of half-height magnetization steps in $\mathrm{Sr}_{2} \mathrm{RuO}_{4}$. Science 331, 186 (2011).

19. Dai, W. et al. Tailoring $\mathrm{LaAlO}_{3} / \mathrm{SrTiO}_{3}$ interface metallicity by oxygen surface adsorbates. Nano Lett. 16, 2739-2743 (2016).

20. Bibes, M., Villlegas, J. E. \& Barthélémy, A. Ultrathin oxide films and interfaces for electronics and spintronics. Adv. Phys. 60, 5 (2011).

21. Lim, H., Jung, J., Ruoff, R. S. \& Kim, Y. Structurally driven one-dimensional electron confinement in sub-5-nm graphene nanowrinkles. Nat. Commun. 6, 8601 (2015).

22. Harrell, R. H., Pyshkin, K. S., Simmons, M. Y. \& Ritchie, D. A. Fabrication of highquality one-and two-dimensional electron gases in undoped GaAs/AlGaAs heterostructures. Appl. Phys. Lett. 74, 2328-2330 (1999).

23. Brus, L. Size, dimensionality, and strong electron correlation in nanoscience. Acc. Chem. Res. 47, 2951 (2014).

24. Pertsev, N. A., Zembilgotov, A. G. \& Tagantsev, A. K. Equilibrium states and phase transitions in epitaxial ferroelectric thin films. Ferroelectrics 223, 79 (1999).

25. Chen, L. et al. Large elasto-optic effect in epitaxial $\mathrm{PbTiO}_{3}$ films. Phys. Rev. Lett. 115, 267602 (2015).

26. Lindman, A., Erhart, P. \& Wahnstrom, G. Polaronic contributions to oxidation and hole conductivity in acceptor-doped $\mathrm{BaZrO}_{3}$. Phys. Rev. B 94, 075204-075213 (2016).

27. Geneste, G., Amadon, B., Torrent, M. \& Dezanneau, G. DFT+U study of selftrapping, trapping, and mobility of oxygen-type hole polarons in barium stannate. Phys. Rev. B 96, 134123 (2017).

28. Erhart, P., Klein, A., Åberg, D. \& Sadigh, B. Efficacy of the DFT+U formalism for modeling hole polarons in perovskite oxides. Phys. Rev. B 90, 035204 (2014).

29. Hao, X., Wang, Z., Schmid, M., Diebold, U. \& Franchini, C. Coexistence of trapped and free excess electrons in $\mathrm{SrTiO}_{3}$. Phys. Rev. B 91, 085204 (2015).

30. Frohlich, H. Interactions of electrons with lattice vibrations. Proc. R. Soc. Lond. Ser. A 215, 291 (1952).

31. Kolodiazhnyi, T., Tachibana, M., Kawaji, H., Hwang, J. \& Takayama-Muromachi, E. Persistence of ferroelectricity in through the insulator-metal transition. Phys. Rev. Lett. 104, 147602 (2010).

32. Wang, Y., Liu, X., Burton, J. D., Jaswal, S. S. \& Tsymbal, E. Y. Ferroelectric instability under screened coulomb interactions. Phys. Rev. Lett. 109, 247601 (2012).

33. Iwazaki, Y., Suzuki, T., Mizuno, Y. \& Tsuneyuki, S. Doping-induced phase transitions in ferroelectric BaTiO from first-principles calculations. Phys. Rev. B 86, 214103 (2012).

34. Li, M., Jia, L., Chen, L. Q., Gu, B. L. \& Duan, W. Effects of strain and oxygen vacancies on the ferroelectric and antiferrodistortive distortions in $\mathrm{PbTiO}_{3} / \mathrm{SrTiO}_{3}$ superlattice. Phys. Rev. B 92, 115435 (2015).

35. Wang, $\mathrm{H}$. et al. Stabilization of highly polar $\mathrm{BiFeO}_{3}$-like structure: a new interface design route for enhanced ferroelectricity in artificial perovskite superlattices. Phys. Rev. X 6, 011027 (2016)

36. King-Smith, R. D. \& Vanderbilt, D. Theory of polarization of crystalline solids. Phys. Rev. B 47, 1651-1654 (1993).

37. Seo, Y.-S., Ahn, J. S. \& Jeong, I.-K. First-principles investigations on polytypes of $\mathrm{BaTiO}_{3}$ : hybrid calculations and pressure dependences. Phys. Soc. 62, 749 (2013).

38. Cohen, R. E. Origin of ferroelectricity in perovskite oxide. Science 358, 136 (1992).

39. Xiang, $\mathrm{H}$. J. Origin of polar distortion in $\mathrm{LiNbO}_{3}$-type "ferroelectric" metals: role of A-site instability and short-range interactions. Phys. Rev. B 90, 094108 (2014).

40. Ye, L. H., Freeman, A. J. \& Delley, B. Half-metallic ferromagnetism in Cu-doped ZnO: density functional calculations. Phys. Rev. B 73, 033203 (2006).

41. Heyd, J., Scuseria, G. E. \& Ernzerhof, M. Hybrid functionals based on a screened coulomb potential. J. Chem. Phys. 118, 8207 (2003)
42. Heyd, J., Scuseria, G. E. \& Ernzerhof, M. Screened hybrid density functionals applied to solids. J. Chem. Phys. 124, 219906 (2006); erratum 124, 154709 (2006)

43. Bidault, O., Maglione, M., Actis, M. \& Kchikech, M. Polaronic relaxation in perovskites. Phys. Rev. B 52, 4191 (1995).

44. Chen, H. \& Umezawa, N. Effect of cation arrangement on the electronic structures of the perovskite solid solutions $\left(\mathrm{SrTiO}_{3}\right)_{1-x}\left(\mathrm{LaCrO}_{3}\right)_{x}$ from first principles. Phys. Rev. B 90, 93-97 (2014).

45. Bertoni, M. I. et al. Tunable conductivity and conduction mechanism in an ultraviolet light activated electronic conductor. J. Appl. Phys. 97, 103713 (2005).

46. Wang, Z. Q., Wu, M. S., Xu, B. \& Ouyang, C. Y. Improving the electrical conductivity and structural sability of the $\mathrm{Li}_{2} \mathrm{MnO}_{3}$ cathode via $\mathrm{P}$ doping. J. Alloy. Compd. 658, 818-823 (2016).

47. Radin, M. D. \& Siegel, D. J. Charge transport in lithium peroxide: relevance for rechargeable metal-air batteries. Energy Environ. Sci. 6, 2370-2379 (2013).

48. Deskins, N. A. \& Dupuis, M. Electron transport via polaron hopping in bulk $\mathrm{TiO}_{2}: \mathrm{a}$ density functional theory characterization. Phys. Rev. B 75, 195212 (2007).

49. Deskins, N. A. \& Dupuis, M. Intrinsic hole migration rates in $\mathrm{TiO}_{2}$ from density functional theory. J. Phys. Chem. C 113, 346-358 (2009).

50. Tuller, H. L. \& Nowick, A. S. Small polaron electron transport in reduced $\mathrm{CeO}_{2}$ single crystals. J. Phys. Chem. Solids 38, 859 (1977).

51. Dieckmann, R., Witt, C. A. \& Mason, T. O. Defects and cation diffusion in magnetite (V): electrical conduction, cation distribution and point defects in $\mathrm{Fe}_{3-\delta} \mathrm{O}_{4}$. Ber. Bunsenges. Phys. Chem. 87, 495 (1983).

52. Bertoni, M. I. et al. Tunable conductivity and conduction mechanism in an ultraviolet light activated electronic conductor. J. Appl. Phys. 97, 103713 (2005).

53. Bertoni, M. I., Mason, T. O., Medvedeva, J. E. \& Wang, Y. Enhanced electronic conductivity in Si-substituted calcium aluminate. J. Appl. Phys. 102, 15 (2007).

54. Nell, J., Wood, B. J., Dorris, S. E. \& Mason, T. O. Jonker-type analysis of small polaron conductors. J. Solid State Chem. 82, 247-254 (1989).

55. Naik, I. K. \& Tien, T. Y. Small-polaron mobility in nonstoichiometric cerium dioxide. J. Phys. Chem. Solids 39, 311-315 (1978).

56. Plata, J. J., Márquez, A. M. \& Sanz, J. F. Electron mobility via polaron hopping in bulk ceria: a first-principles study. J. Phys. Chem. C 117, 14502-14509 (2013).

57. Zheng, P., Zhang, R. Z., Chen, H. Y. \& Hao, W. T. Thermoelectric properties and conduction mechanism of $\mathrm{CaCu}_{3} \mathrm{Ti}_{4} \mathrm{O}_{12}$ ceramics at high temperatures. J. Electron. Mater. 43, 1645-1649 (2014).

58. Liu, Y., Qin, X. Y., Xin, H. X., Zhang, J. \& Li, H. J. Electrical transport and thermoelectric properties of $\mathrm{Y}_{1-x} \mathrm{Ca}_{x} \mathrm{CoO}_{3}(0 \leq x \leq 0.1)$ at high temperatures. J. Appl. Phys. 101, 083709 (2007).

59. Karim, D. P. \& Aldred, A. T. Localized level hopping transport in $\mathrm{La}(\mathrm{Sr}) \mathrm{CrO}_{3}$. Phys. Rev. B 20, 2255 (1979).

60. Ohtaki, M., Tokunaga, T., Eguchi, K., Arayi, H. Proceedings of the 16th International onference on Thermoelectrics IEEE, p. 224 (1997).

61. Kohmoto, T., Ikeda, D., Liang, X. \& Moriyasu, T. Direct observation of the spatial and temporal dynamics of polaron diffusion in $\mathrm{SrTiO}_{3}$. Phys. Rev. B 87, 214301 (2013).

62. Ueno, K. et al. Electric-field-induced superconductivity in an insulator. Nat. Mater. 7, 855-858 (2008)

63. Kolodiazhnyi, T., Tachibana, M., Kawaji, H., Hwang, J. \& Takayama-Muromachi, E. Persistence of ferroelectricity in $\mathrm{BaTiO}_{3}$ through the insulator-metal transition. Phys. Rev. Lett. 104, 147602 (2010).

64. Wang, Y., Liu, X., Burton, J. D., Jaswal, S. S. \& Tsymbal, E. Y. Ferroelectric instability under screened coulomb interactions. Phys. Rev. Lett. 109, 247601 (2012).

65. Zhao, H. J. et al. Meta-screening and permanence of polar distortion in metallized ferroelectrics. Phys. Rev. B 97, 054107 (2018).

66. Paillard, C., Geneste, G., Bellaiche, L. \& Dkhil, B. Vacancies and holes in bulk and at $180^{\circ}$ domain walls in lead titanate. J. Phys. Condens. Matter 29, 485707 (2017).

67. Anisimov, V. I., Zaanen, J. \& Andersen, O. K. Band theory and Mott insulators: Hubbard U instead of Stoner I. Phys. Rev. B 44, 943 (1991).

68. Kresse, G. \& Hafner, J. Ab initio molecular dynamics for liquid metals. Phys. Rev. $B$ 47, 558 (1993).

69. Kresse, G. \& Furthmüller, J. Efficient iterative schemes for ab initio total-energy calculations using a plane-wave basis set. Phys. Rev. B 54, 11169 (1996).

70. Monkhorst, H. J. \& Pack, J. D. Special points for Brillouin-zone integrations. Phys. Rev. B 13, 5188 (1976).

71. Aryasetiawan, F., Karlsson, K., Jepsen, O. \& Schonberger, U. Calculations of Hubbard U from first-principles. Phys. Rev. B 74, 125106 (2006).

72. Setvin, M. et al. Direct view at excess electrons in rutile and anatase. Phys. Rev. Lett. 113, 086402 (2014).

73. Makov, G. \& Payne, M. C. Periodic boundary conditions in ab initio calculations. Phys. Rev. B 51, 4014 (1995)

74. Farazdel, A., Dupuis, M., Clementi, E. \& Aviram, A. Electric-field induced intramolecular electron transfer in spiro pi-electron systems and their suitability as molecular electronic devices. A theoretical study. J. Am. Chem. Soc. 112, 4206 (1990). 
75. Deskins, N. A. \& Dupuis, M. Electron transport via polaron hopping in bulk $\mathrm{TiO}_{2}$ : a density functional theory characterization. Phys. Rev. B 75, 195212 (2007).

76. Bertoni, M. I. et al. Enhanced electronic conductivity in Si-substituted calcium aluminate. J. Appl. Phys. 102, 113704 (2007).

(c) (i) Open Access This article is licensed under a Creative Commons Attribution 4.0 International License, which permits use, sharing, adaptation, distribution and reproduction in any medium or format, as long as you give appropriate credit to the original author(s) and the source, provide a link to the Creative
Commons license, and indicate if changes were made. The images or other third party material in this article are included in the article's Creative Commons license, unless indicated otherwise in a credit line to the material. If material is not included in the article's Creative Commons license and your intended use is not permitted by statutory regulation or exceeds the permitted use, you will need to obtain permission directly from the copyright holder. To view a copy of this license, visit http://creativecommons. org/licenses/by/4.0/.

(c) The Author(s) 2019 